\title{
Rural-urban disparity in category II vaccination among children under five years of age: evidence from a survey in Shandong, China
}

\author{
Xinyi Zhang ${ }^{1}$, Zerin Imam Syeda', Zhengyue Jing ${ }^{1}$, Qiongqiong X ${ }^{1}$, Long Sun ${ }^{1,2}$, Lingzhong Xu \\ and Chengchao Zhou ${ }^{1,2^{*}}$ (D)
}

\begin{abstract}
Background: Compared with the Expanded Program on Immunization (EPI) vaccines, the coverage rate of the non-EPI vaccines is still low. The aim of this study is to explore the rural-urban disparity in category II vaccine and its determinants among children under 5 years old in China.

Methods: A cross-sectional study was conducted in 17 cities in Shandong province from August to October, 2013. A total of 1638 children were included in the analysis. Unadjusted and adjusted regression model were used to identify the rural-urban difference in vaccination of category II vaccine. Multivariate logistic regression models were employed to analyze the determinants associated with vaccination of category $\|$ vaccine in rural and urban areas respectively.

Results: The coverage rates of category II vaccine in rural and urban children were 81.5 and $69.4 \%$ respectively. Factors including age and satisfaction with vaccination services were associated with category II vaccination both in rural and urban children $(P<0.05)$. It was also found that the households with four or less members are more likely to vaccinate category $\|$ vaccine in rural children.

Conclusions: There was a big difference between rural and urban children in the use of category II vaccine. The government should strengthen financial support and regulation for the category II vaccine. The identified at-risk factors, including age, satisfaction with the vaccination services, and family size should be taken into account when designing targeted vaccination policies for rural and urban children.
\end{abstract}

\section{Background}

Vaccine immunization is a highly cost-effective public health intervention which has played an important role in preventing children from infectious diseases [1]. According to the World Health Organization (WHO) estimates in 2012, immunization can prevent 2 to 3 million deaths each year [2]. Furthermore, among the most cost-effective measures to address the xpanded children's immunization coverage ranked third, at the Copenhagen Consensuworld's major challenges, es Center in 2012 [3]. China established

\footnotetext{
* Correspondence: zhouchengchao@sdu.edu.cn

${ }^{1}$ School of Public Health, Shandong University, Jinan 250012, China

${ }^{2}$ Key Lab of Health Economics and Policy Research, Shandong University, 44 Wen-hua-xi Road, Jinan 250012, Shandong, China
}

the expanded program on immunization (EPI) in 1978. In 2007, China implement the national EPI, the type of vaccine-preventable diseases also expanded from 6 to15 [4]. The EPI vaccines are provided free of charge by the government, while non-EPI vaccine, which is also called category II vaccine or self-paid vaccine, is a voluntary vaccine by the citizens at their own expense [5].

Globally, vaccine-preventable disease is a serious public health burden [6]. China has a large birth cohort, so the category II vaccine such as $\mathrm{H}$. Influenza type b (HIB) and S. pneumoniae etc. corresponding diseases are more worthy of attention. The WHO estimates that China's S. pneumoniae infections and deaths accounted for 12 and $3.6 \%$ of the global burden, and HIB infections and deaths

(C) The Author(s). 2018 Open Access This article is distributed under the terms of the Creative Commons Attribution 4.0 International License (http://creativecommons.org/licenses/by/4.0/), which permits unrestricted use, distribution, and 
Table 1 Socio-demographic characteristics of study participants in Shandong, China, 2013

\begin{tabular}{|c|c|c|c|c|}
\hline Variable & Overall & Urban & Rural & ${ }^{*} P$-value \\
\hline$N(\%)$ & $1638(100)$ & 764 & 874 & \\
\hline Sex & & & & 0.136 \\
\hline Boy & $894(54.6)$ & $402(52.6)$ & $492(56.3)$ & \\
\hline Girl & $744(45.4)$ & $362(47.4)$ & $382(43.7)$ & \\
\hline Age & & & & 0.714 \\
\hline $0-1$ & $500(30.5)$ & 240(31.4) & $260(29.7)$ & \\
\hline-2 & $320(19.5)$ & 149(19.5) & $171(19.6)$ & \\
\hline-3 & 350(21.4) & $169(22.1)$ & $181(20.7)$ & \\
\hline-4 & $303(18.5)$ & $132(17.3)$ & $171(19.6)$ & \\
\hline-5 & 165(10.1) & $74(9.7 \%)$ & $91(10.4)$ & \\
\hline Family size & & & & 0.241 \\
\hline$\leq 4$ & $916(55.9)$ & $439(57.5)$ & $477(54.6)$ & \\
\hline$>4$ & $722(44.1)$ & $325(42.5)$ & $397(45.4)$ & \\
\hline Household income ${ }^{\text {a }}$ & & & & 0.000 \\
\hline Q1 & $363(22.2)$ & $121(15.8)$ & $242(27.7)$ & \\
\hline Q2 & $292(17.8)$ & $117(15.4)$ & 175(20.0) & \\
\hline Q3 & $333(20.3)$ & $149(19.5)$ & 184(21.1) & \\
\hline Q4 & $364(22.2)$ & $186(24.3)$ & $178(20.4)$ & \\
\hline Q5 & $286(17.5)$ & $191(25.0)$ & $95(10.9)$ & \\
\hline Physical examination & & & & 0.320 \\
\hline Yes & $988(60.3)$ & $451(59.0)$ & $537(61.4)$ & \\
\hline No & $650(39.7)$ & $313(41.0)$ & $337(28.6)$ & \\
\hline Primary caregiver & & & & 0.000 \\
\hline Parents & 1445(88.2) & 700(91.6) & $745(85.2)$ & \\
\hline Grandparents & 193(11.8) & $64(8.4)$ & $129(14.8)$ & \\
\hline Primary caregiver's education & & & & 0.000 \\
\hline Primary and below & $247(15.1)$ & $44(5.8)$ & 203(23.2) & \\
\hline Junior education & $821(50.1)$ & $320(41.9)$ & $501(57.3)$ & \\
\hline Senior and above & $570(34.8)$ & $400(52.4)$ & $170(19.5)$ & \\
\hline Satisfaction ${ }^{b}$ & & & & 0.496 \\
\hline Satisfaction & 1396(85.2) & 656(85.9) & $740(84.7)$ & \\
\hline Non-satisfaction & $242(14.8)$ & $108(14.1)$ & $134(15.3)$ & \\
\hline
\end{tabular}

Note: The $P$-values indicate statistical significance at $5 \%$ level, the italics indicate significance

${ }^{a}$ Quintile $1(\mathrm{Q} 1)$ is the poorest and Quintile $5(\mathrm{Q} 5)$ is the richest *: Chi-test

${ }^{\mathrm{b}}$ Satisfaction with the vaccination services

accounted for 14 and $5.1 \%$ of the global burden, respectively [7, 8]. Many studies have demonstrated that expanding coverage of category II vaccine has a highly cost-effectiveness [9-11].

However, in China, the actual vaccination rate of category II vaccines is remarkably different from those of EPI vaccines and inoculation rate varies widely in different regions. Previous studies have demonstrated that the coverage of category II vaccines in children aged $1 \sim 2$ was $61.42 \%$ in China, while this proportion was $82.51,64.86$ and $43.18 \%$ in eastern, central and western China respectively [12].Other studies have also demonstrated that there was a difference in the utilization of category II vaccines among families with different socio-economic status [1, 13-15].

Most of the existing studies only focused on the associated factors and vaccination rates for category II vaccine [16-19].To date, no studies have examined the difference in the rates of category II vaccination in children between urban and rural China. In this study, we aim to explore the difference in the use of category II vaccine between rural and urban China. To do so, we have the following specific objectives. First, we will compare the use of category II vaccine between rural and urban children in China. Second, we will identify the factors associated with the use of category II vaccine in rural and urban children respectively. 


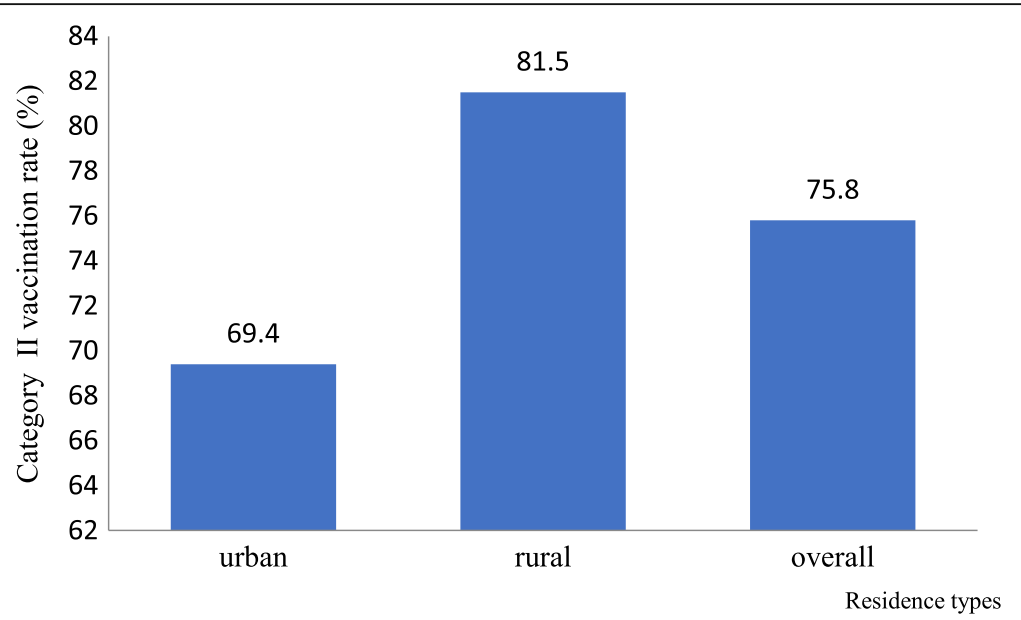

Fig. 1 Category II vaccination rate of children among different groups in Shandong, China, 2013

\section{Methods}

\section{Data source}

Data used in this study were obtained from the 5th National Health Service Surveys (NHSS) of Shandong conducted in 2013. NHSS is a nationally representative survey of China organized and directed by the National Health and Family Planning Commission of China every 5 year. Shandong is the second largest province in China and its children (0-14) account for $16.1 \%$ of the total population (about 97million) [20].According to previous studies, category II vaccination rate among children under 5 years old was significantly lower than that of EPI vaccines $[21,22]$. In this study, a four-stage, stratified cluster sampling was used to select participants. First, we randomly selected 20 counties (urban districts) from all prefectural cities in Shandong province, and in each county (district), we selected 5 townships (sub-districts). Second, we chose two villages (communities) from the each selected township (sub-district) randomly. Thus, 100 township (sub-districts) and 200 villages (communities) were chosen as study sites. Third, 60 children were randomly selected from each sample village (community) and the youngest one in each household was investigated. Eventually, a total of 1638 children were included in the analysis, of which the number of urban children was 764, and rural children was 874 .

\section{Variables and measures \\ Dependent variable}

The dependent variable was vaccination of category II vaccines in children under 5 years old, which was assessed on the ground of primary guardians' answers to - "Has your child been vaccinated with the following category II or self-paid vaccines (Haemophilus influenzae type b vaccine, Pneumococcal polysaccharide vaccine, Varicella attenuated live vaccine, Influenza vaccine and Measles and mumps combined attenuated live vaccine or others)?" As long as vaccinate any one type or more of the category II vaccines, it was classified as "yes". The difference between category I and category II vaccines lies in that the category I vaccines are provided free of charge by the government, but the category II vaccines are paid fully by the users themselves. When conducting the interview, the vaccination certificate of the interviewed child would be also reviewed by the interviewers to confirm the response of the interviewees.

\section{Independent variable}

Socio-demographic characteristics, which including gender (boy versus girl), age $(0-1,-2,-3,-4,-5$ years), family size $(\leq 4$ versus $>4)$, primary caregiver (grandparents versus parents), primary caregivers' education attainment (primary and below, junior school, senior school and above), physical examination (yes vs. no), satisfaction (satisfaction vs. non-satisfaction), and household income (Q1, Q2, Q3, Q4 and Q5).

"Physical examination" was collected by asking the children's primary caregivers "How many times physical examinations have your child had received in the past 12 months (Excluding those examinations for the diagnosis or treatment for some special diseases)?" If the answer was "once or more", we coded it as "yes"; and if the answer was "none", we coded it as "no".

As for "Satisfaction", we collected the data by asking the primary caregivers a question of "Are you satisfied with the vaccination services the health care facilities provide for your child?" . If the answer was "yes", we coded it as "yes"; and if the answer were "normal" or "no", we coded it as "no".

When measuring "household income", we used a question of "How much was the total income of your family in the last year? (Urban households were disposable income, rural households were net income)" It was divided 
into the same group distance: $20,40,60$, and $80 \%$, based on the total income of households in the last year from highest to lowest, the income quintiles can better reflect the grouping of resident's income. Quintile 1 (Q1) was the poorest and Quintile 5 (Q5) was the richest.

\section{Statistical analysis}

The data were double entered using EpiData 6.04. Statistical analyses were performed using SPSS 16.0. Chi-square tests were employed to evaluate socio-demographic differences between urban and rural children. Two models, one unadjusted and one adjusted regression model were used to identify the rural-urban difference in vaccination of category II vaccines. Multivariate logistic regression model was employed to analyze relevant factors associated with vaccination of category II vaccines in rural and urban areas respectively. Sampling weights were included in all analyses to adjust for the complex survey design. Statistical significance was set at the $5 \%$ level.

\section{Results}

\section{Socio-demographic characteristics}

A total of 1638 children and their primary caregivers participated in this study, of which urban children accounted for $46.6 \%$ (764), and rural children accounted for $53.4 \%$ (874). There were no significant differences in gender, age, family size, physical examination, and satisfaction between urban and rural children. Generally speaking, the probability of urban children lived in higher-income households as higher than that in rural children $(P=0.000)$. Among the primary caregivers of urban children, the proportion of parents is higher $(P=0.000)$ than that of rural children, and the primary caregivers also had significant higher education attainment in urban children $(P=0.000)$ than that in rural children (Table 1).

\section{Category II vaccination rate}

Category II vaccination rate of children among different groups is shown in Fig. 1. We can clearly see that the overall rate of category II vaccines was $75.8 \%$ (1242/1638). When we compared the category II vaccination rate between urban and rural children, we found that the rate of category II vaccination was significantly higher in rural children (81.5\%) than that in urban children (69.4\%).

\section{Category II vaccination status in urban and rural areas}

We used two models, one unadjusted and one adjusted logistic regression model, to assess the association between residence and category II vaccination status in children under 5 years old (Table 2). The unadjusted model showed that the rate of category II vaccines in rural children was statistically higher than that in urban children $(P=0.000)$. When we controlled for other
Table 2 Association of category II vaccination status with residence types in Shandong, China

\begin{tabular}{|c|c|c|c|c|c|c|}
\hline \multirow[t]{2}{*}{ Variable } & \multicolumn{3}{|c|}{ Model 1 (No covariates) } & \multicolumn{3}{|c|}{ Model 2 (covariates) } \\
\hline & $\bar{P}$ & OR & $\overline{\mathrm{OR} 95 \% \mathrm{Cl}}$ & $\bar{P}$ & OR & $\overline{\mathrm{OR} 95 \% \mathrm{Cl}}$ \\
\hline \multicolumn{7}{|l|}{$\overline{\text { Area }}$} \\
\hline Urban & & 1.0 & & & 1.0 & \\
\hline Rural & 0.000 & 1.94 & $1.54-2.44$ & 0.000 & 1.93 & $1.48-2.52$ \\
\hline \multicolumn{7}{|l|}{ Sex } \\
\hline Boy & & & & & 1.0 & \\
\hline Girl & & & & 0.829 & 1.03 & $0.81-1.31$ \\
\hline \multicolumn{7}{|l|}{ Age } \\
\hline-5 & & & & & 1.0 & \\
\hline $0-1$ & & & & 0.000 & 0.29 & $0.18-0.46$ \\
\hline-2 & & & & 0.575 & 0.87 & $0.52-1.43$ \\
\hline-3 & & & & 0.457 & 1.22 & $0.73-2.04$ \\
\hline-4 & & & & 0.738 & 1.09 & $0.65-1.84$ \\
\hline \multicolumn{7}{|l|}{ Family size } \\
\hline$>4$ & & & & & 1.0 & \\
\hline$\leq 4$ & & & & 0.443 & 1.10 & $0.86-1.41$ \\
\hline \multicolumn{7}{|l|}{ Household income } \\
\hline $\mathrm{Q}^{\mathrm{a}}$ & & & & & 1.0 & \\
\hline Q1 & & & & 0.026 & 0.64 & $0.43-0.95$ \\
\hline Q2 & & & & 0.448 & 0.86 & $0.57-1.31$ \\
\hline Q3 & & & & 0.339 & 1.23 & $0.81-1.86$ \\
\hline Q4 & & & & 0.861 & 0.97 & $0.66-1.42$ \\
\hline \multicolumn{7}{|l|}{ Physical examination } \\
\hline No & & & & & 1.0 & \\
\hline Yes & & & & 0.479 & 1.09 & $0.85-1.41$ \\
\hline \multicolumn{7}{|l|}{ Primary caregiver } \\
\hline Grandparents & & & & & 1.0 & \\
\hline Parents & & & & 0.497 & 1.14 & $0.85-1.41$ \\
\hline \multicolumn{7}{|c|}{ Primary caregiver's education } \\
\hline Primary and below & & & & & 1.0 & \\
\hline Junior education & & & & 0.202 & 1.28 & $0.88-1.88$ \\
\hline Senior and above & & & & 0.411 & 0.84 & $0.55-1.27$ \\
\hline \multicolumn{7}{|l|}{ Satisfaction ${ }^{b}$} \\
\hline Satisfaction & & & & & 1.0 & \\
\hline Non-satisfaction & & & & 0.000 & 1.87 & $1.37-2.59$ \\
\hline
\end{tabular}

Note: The P-values indicate statistical significance at $5 \%$ level, the italics indicate significance

${ }^{a}$ Quintile $1(\mathrm{Q} 1)$ is the poorest and Quintile $5(\mathrm{Q} 5)$ is the richest

${ }^{\mathrm{b}}$ Satisfaction with the vaccination services

variables, compared with urban children, vaccination rate was still higher in rural children $(P=0.000)$.

Determinants of category II vaccination status among urban and rural children

Univariate analysis was conducted to identify the factors independently associated with category II vaccination status in rural children (Table 3) and urban children (Table 4) respectively. Two factors were found to be 
Table 3 Factors associated with category II vaccination status of children in urban Shandong, China, 2013

\begin{tabular}{|c|c|c|c|c|c|c|c|c|}
\hline Variable & Vaccinated (\%) & Unvaccinated (\%) & $P$ & ORC & ORc95\%Cl & $P$ & $\mathrm{ORa}^{*}$ & ORa95\%C \\
\hline$n=764$ & $530(69.4)$ & 234(30.6) & & & & & & \\
\hline Sex & & & & & & $N A^{*}$ & & \\
\hline Boy & $279(69.4)$ & 123(30.6) & & 1.0 & & & & \\
\hline Girl & $251(69.3)$ & $111(30.7)$ & 0.984 & 0.99 & $0.73-1.36$ & & & \\
\hline \multicolumn{9}{|l|}{ Age } \\
\hline-5 & $60(81.1)$ & 14(18.9) & & 1.0 & & & 1.0 & \\
\hline $0-1$ & $111(46.3)$ & $129(53.7)$ & 0.000 & 0.20 & $0.11-0.38$ & 0.000 & 0.21 & $0.11-0.39$ \\
\hline-2 & $114(76.5)$ & $35(23.5)$ & 0.438 & 0.76 & $0.38-1.52$ & 0.485 & 0.78 & $0.38-1.58$ \\
\hline-3 & 135(79.9) & $34(20.1)$ & 0.829 & 0.93 & $0.46-1.85$ & 0.873 & 0.94 & $0.47-1.92$ \\
\hline-4 & $110(83.3)$ & $22(16.7)$ & 0.683 & 1.17 & $0.56-2.45$ & 0.433 & 1.36 & $0.63-2.90$ \\
\hline Family size & & & & & & NA & & \\
\hline$>4$ & $232(71.4)$ & $93(38.6)$ & & 1.0 & & & & \\
\hline$\leq 4$ & 298(67.9) & $141(32.1)$ & 0.299 & 0.85 & $0.62-1.16$ & & & \\
\hline Household income & & & & & & NA & & \\
\hline $\mathrm{Q} 5^{\mathrm{a}}$ & 136(71.2) & $55(28.8)$ & & 1.0 & & & & \\
\hline Q1 & $79(65.3)$ & $42(34.7)$ & 0.272 & 0.76 & $0.47-1.24$ & & & \\
\hline Q2 & $78(66.7)$ & $39(33.3)$ & 0.402 & 0.81 & $0.49-1.33$ & & & \\
\hline Q3 & $115(77.2)$ & $34(22.8)$ & 0.214 & 1.73 & $0.83-2.24$ & & & \\
\hline Q4 & $122(66.6)$ & 64(33.4) & 0.242 & 0.77 & $0.50-1.19$ & & & \\
\hline Physical examination & & & & & & NA & & \\
\hline No & 225(71.9) & $88(28.1)$ & & 1.0 & & & & \\
\hline Yes & $305(67.6)$ & $146(32.4)$ & 0.210 & 0.82 & $0.59-1.12$ & & & \\
\hline Primary caregiver & & & & & & NA & & \\
\hline Grandparents & $42(65.6)$ & $22(34.4)$ & & 1.0 & & & & \\
\hline Parents & $488(69.7)$ & 212(30.3) & 0.497 & 1.21 & $0.70-2.07$ & & & \\
\hline \multicolumn{9}{|l|}{ Satisfaction b } \\
\hline Satisfaction & $62(57.4)$ & $46(42.6)$ & & 1.0 & & & 1.0 & \\
\hline Non-satisfaction & $468(71.3)$ & $188(28.7)$ & 0.004 & 1.85 & $1.22-2.81$ & 0.003 & 2.02 & $1 . .26-3.22$ \\
\hline \multicolumn{6}{|c|}{ Primary caregiver's education attainment } & NA & & \\
\hline Primary and below & $32(72.7)$ & $12(27.3)$ & & 1.0 & & & & \\
\hline Junior education & $242(75.6)$ & $78(24.4)$ & 0.676 & 1.16 & $0.57-2.37$ & & & \\
\hline Senior and above & $256(64)$ & 144(36) & 0.252 & 0.67 & $0.33-1.34$ & & & \\
\hline
\end{tabular}

Note: The P-values indicate statistical significance at $5 \%$ level, the italics indicate significance

"ORc Crude odds ratio, ${ }^{*}$ ORa Adjusted odds ratio, "NA Not applicable

${ }^{a}$ Quintile $1(\mathrm{Q} 1)$ is the poorest and Quintile $5(\mathrm{Q} 5)$ is the richest

${ }^{\mathrm{b}}$ Satisfaction with the vaccination services

determinants $(P<0.05)$ both in rural and urban children, including age and satisfaction. Compared with urban children, children in rural areas which with less than four family members tended to use category II vaccines $(P<0.05)$. We also observed the same results in multivariate logistic regression models for the rural and urban children $(P<0.05)$.

\section{Discussion}

The present study explores, for the first time, the rural-urban difference in category II vaccination of children under 5 years in China. This study shows that the overall rate of category II vaccines for children aged $0-5$ is $75.8 \%$ in Shandong province. The rate of category II vaccines is lower than the $88.24 \%$ found in Jiangdong district, China [23]. It's also a little lower than the rate of $78.31 \%$ in the children aged 2 to 6 years in Tianjin [24]. However, it is higher than the inoculation rate of category II vaccines $(61.42,73.24 \%)$ in some other studies in China [12, 25]. Compared with economically developed regions in China, there is still a little gap in the category II vaccination rate in Shandong province.

Contrary to previous studies in China $[17,26]$, our results show that category II vaccines rate in urban children is significantly lower than that in rural children. 
Table 4 Factors associated with category II vaccination status of children in rural Shandong, China, 2013

\begin{tabular}{|c|c|c|c|c|c|c|c|c|}
\hline Variable & Vaccinated (\%) & Unvaccinated (\%) & $P$ & $O R c^{*}$ & ORc95\%Cl & $P$ & $\mathrm{ORa}^{*}$ & ORa95\%Cl \\
\hline$n=874$ & $712(81.5)$ & $162(18.5)$ & & & & & & \\
\hline Sex & & & & & & $N A^{*}$ & & \\
\hline Boy & 400(81.3) & $92(18.7)$ & & 1.0 & & & & \\
\hline Girl & $312(81.7)$ & $70(18.3)$ & 0.888 & 1.03 & $0.73-1.45$ & & & \\
\hline \multicolumn{9}{|l|}{ Age } \\
\hline-5 & 77(84.6) & $14(15.4)$ & & 1.0 & & & 1.0 & \\
\hline $0-1$ & 184(70.8) & $76(29.2)$ & 0.011 & 0.44 & $0.24-0.83$ & 0.003 & 0.36 & $0.19-0.71$ \\
\hline-2 & $144(84.2)$ & $27(15.8)$ & 0.932 & 0.97 & $0.48-1.96$ & 0.681 & 0.86 & $0.42-1.77$ \\
\hline-3 & 164(90.6) & $17(9.4)$ & 0.146 & 1.75 & $0.82-3.74$ & 0.285 & 1.53 & $0.71-3.32$ \\
\hline-4 & 143(83.6) & $28(16.4)$ & 0.835 & 0.93 & $0.46-1.87$ & 0.632 & 0.84 & $0.41-1.73$ \\
\hline \multicolumn{9}{|l|}{ Family size } \\
\hline$>4$ & $311(78.3)$ & $86(21.7)$ & & 1.0 & & & 1.0 & \\
\hline$\leq 4$ & $401(84.1)$ & 76(15.9) & 0.031 & 1.46 & $1.04-2.06$ & 0.010 & 1.62 & $1.12-2.33$ \\
\hline Household income & & & & & & NA & & \\
\hline $\mathrm{Q}^{\mathrm{a}}$ & $77(81.1)$ & 18(18.9) & & 1.0 & & & & \\
\hline Q1 & 181(74.8) & $61(25.2)$ & 0.224 & 0.69 & $0.39-1.25$ & & & \\
\hline Q2 & 143(81.7) & $32(18.3)$ & 0.894 & 1.05 & $0.55-1.98$ & & & \\
\hline Q3 & 157(85.3) & $27(14.7)$ & 0.359 & 1.36 & $0.71-2.62$ & & & \\
\hline Q4 & 154(86.5) & $24(13.5)$ & 0.235 & 1.50 & $0.77-2.93$ & & & \\
\hline Physical examination & & & & & & NA & & \\
\hline No & 269(79.8) & $68(20.2)$ & & 1.0 & & & & \\
\hline Yes & $443(82.5)$ & $94(17.5)$ & 0.323 & 1.19 & $0.84-1.69$ & & & \\
\hline Primary caregiver & & & & & & NA & & \\
\hline Grandparents & 105(81.4) & 24(18.6) & & 1.0 & & & & \\
\hline Parents & $607(81.5)$ & 138(18.5) & 0.983 & 1.01 & $0.62-1.63$ & & & \\
\hline Satisfaction ${ }^{\mathrm{b}}$ & & & & & & NA & & \\
\hline Satisfaction & 96(71.6) & $38(28.4)$ & & 1.0 & & & 1.0 & \\
\hline Non-satisfaction & 616(83.2) & $124(16.8)$ & 0.002 & 1.97 & $1.29-3.00$ & 0.007 & 1.87 & $1.19-2.93$ \\
\hline Primary caregiver's education attainment & & & & & & NA & & \\
\hline Primary and below & 162(79.8) & $41(10.2)$ & & 1.0 & & & & \\
\hline Junior education & $416(83.0)$ & $85(17.0)$ & 0.312 & 1.24 & $0.53-1.22$ & & & \\
\hline Senior and above & 134(78.8) & $36(21.2)$ & 0.816 & 0.94 & $0.57-1.56$ & & & \\
\hline
\end{tabular}

Note: The P-values indicate statistical significance at $5 \%$ level, the italics indicate significance

*ORc Crude odds ratio, ${ }^{*}$ ORa Adjusted odds ratio, "NA Not applicable

${ }^{a}$ Quintile $1(\mathrm{Q} 1)$ is the poorest and Quintile $5(\mathrm{Q} 5)$ is the richest

${ }^{\mathrm{b}}$ Satisfaction with the vaccination services

Different from the unified national policy for the EPI vaccines, the current provision of category II vaccines mainly depends on the grassroots' disease prevention and control units and even vaccination stations [27]. In rural areas, primary health providers usually have close connection with residents. Therefore, the primary caregivers of rural children are better encouraged to vaccinate, with the efforts of rural health providers [28].Secondly, it is found in previous studies that perceived social norms were positively correlated with willingness to pay [29]. The social impact of the group effect is an important factor in the decision-making of the child's vaccination. This group effect is more obvious in the rural areas where the population is closely linked. This is also consistent with the findings in other countries [30-32].Thirdly, vaccination safety has always been a concern of the guardians, and any negative news of vaccination is likely to cause short or long-term effects on vaccination use. Many guardians are afraid of the vaccination insecurity or fear of adverse reactions, as a 
result they would not use vaccination [27, 33]. In the rural areas, where the influence of mass media is limited, this negative effect will be relatively low [34]. The above reasons might explain the rural-urban difference in category II vaccination use found in the current study.

Economic status was found to be a determinant for category II vaccines use, which was similar with previous studies [17-19]. Ideally, the health service system should ensure equal access to public health services, regardless of gender, residence and capacity to pay. In the current study, compared with high-income households, lower income ones have a lower probability of vaccination. One possible reason is that vaccine pricing and funding mechanisms have a significant impact on children's vaccination. Currently, there is no uniform pricing standard for category II vaccines in China. This provides the possibility for healthcare providers to raise the price of the vaccines. The study conducted in 2013 on the willingness of category II vaccines indicated that the procurement price up to 15 and $43 \%$ for county CDC (Center for Disease Prevention and Control) and vaccination clinics, respectively [35], and this price elasticity would probably affect the demand for vaccination, especially in low-income households. Another possible reason might be that, unlike the EPI vaccines with the government financial subsidies, the cost of the category II vaccine is entirely paid by the vaccine recipients. Such cost, in most cases, might adversely affect the use of category II vaccines. This finding indicates a need for the government to regulate the management of category II vaccines, so as to ensure the rationality and transparency of vaccine prices. In addition, financing subsidies should also be expanded to reduce the cost of category II vaccines inoculation, especially for those low-income households.

Among the socio-economic factors, age and satisfaction for the vaccination services are significantly associated with the category II vaccination use both in rural and urban children. Our study demonstrates that those children, who are older, whose primary caregivers are satisfied with immunization institutions' service are more likely to vaccinate category II vaccines. Many previous studies have found that vaccination service providers play an important role in caregivers' vaccination decisions, and caregivers' satisfaction with vaccination is positively related to immunization [36, 37]. The results also show that the households with four or less members are more likely to vaccinate category II vaccines in rural children. One possible explanation might be that the number of children in a small family may be less, and in such families the caregivers might have more money to use category II vaccines for their children, compared with those families with more children [38-40].

Interestingly, the current study shows that there is no gender difference in vaccination rate both in rural and urban, which has also been found in previous studies $[41,42]$. This might be due to that gender discrimination has never been a problem in China. Consistent with previous studies [26, 40], our study also shows that primary caregiver's education attainment is not associated with vaccination rate.

This study had some limitations. First, the data used in this study were derived from a cross-sectional design, the associations between identified factors and category II vaccination cannot be interpreted as cause and effect. Second, many of the variables in our questionnaire were measured by self-reported, which might result in several kinds of bias, such as recall bias.

\section{Conclusions}

This study finds that there is a significant gap in category II vaccine use in children under 5 years between urban (69.4\%) and rural (81.5\%) China. The factors including age and satisfaction for the vaccination services are found to be associated with category II vaccination use both in rural and urban areas. In addition, economic status is still a determinant for category II vaccine. The government should strengthen financial support and regulation for the category II vaccine. The identified at-risk factors, including age, satisfaction with the vaccination services, and family size should be taken into account when designing targeted vaccination policies for rural and urban children.

\section{Abbreviations}

EPI: Expanded program on immunization; HIB: H. influenzae type b.; NHSS: National Health Service Surveys

\section{Acknowledgments}

We thank the officials of local health agencies, all participants and staffs at the study sites for their cooperation.

\section{Funding}

We are grateful for funding support from the National Science Foundation of China (71003067, 71473152 and 71774104), Chinese Medical Board (CMB-16257), Cheeloo Youth Scholar Grant, and Shandong University (IFYT1810, 2012DX006,).

\section{Availability of data and materials}

Please contact the corresponding author for data requests.

\section{Authors' contributions}

CZ, XZ conceived the idea. CZ, XZ, ZJ, QX, and LS participated in the statistical analysis and interpretation of the results. ZIS and LX gave many valuable comments on the draft and also polished it. All authors read and approved the final manuscript.

\section{Ethics approval and consent to participate}

The study protocol was approved by The Ethical Committee of Shandong University School of Public Health. The investigation was performed after the acquisition of informed consents of all participants.

Consent for publication

Not applicable.

Competing interests

The authors declare that they have no competing interests. 


\section{Publisher's Note}

Springer Nature remains neutral with regard to jurisdictional claims in published maps and institutional affiliations.

Received: 19 December 2017 Accepted: 12 June 2018

Published online: 22 June 2018

\section{References}

1. Ganczak M, Dmytrzyk-Daniłów G, Karakiewicz B, et al. Determinants influencing self-paid vaccination coverage, in $0-5$ years old polish children. Vaccine. 2013:31(48):5687-92.

2. Virot P. 10 facts on immunization (World Health Organization). 2012.

3. Copenhagen Consensus. Expert Panel Findings. 2012. Available: http://www. copenhagenconsensus.com/Proje?cts/CC12/Outcome.aspx. Accessed 15 June 2018.

4. Ministry of Health. Expanding the national immunization plan and implement program. Chin Vaccin Immunization. 2007;4(2):183-4.

5. State Council, P.R. China regulation on vaccine circulation and immunization management. Health policy: health policy and regulations, the State Council regulations, 2005

6. Centers for Disease Control and Prevention (CDC). Impact of vaccines universally recommended for children-United States, 1990-1998. MMWR Morb Mortal Wkly Rep. 1999;48(12):243.

7. O'Brien KL, Wolfson LJ, Watt JP, et al. Burden of disease caused by Streptococcus pneumoniae in children younger than 5 years: global estimates. Lancet. 2009:374(9693):893-902

8. Watt JP, Wolfson LJ, O'Brien KL, et al. Burden of disease caused by Haemophilus influenzae type $b$ in children younger than 5 years: global estimates. Lancet. 2009;374(9693):903-11.

9. Liu G, Zhu L, Shen D, et al. PIH22 cost effectiveness of an infant pneumococcal conjugate vaccine program in China. Value Health. 2011; 14(7):A401.

10. Fu Y, Liu G, Zhu L, et al. Cost-effectiveness analysis of seven-valent pneumococcal conjugate vaccine against pneumococcal disease. China Health Econ. 2013;32(1):49-52.

11. Zhu L, Liu G, Li DM, et al. Cost - effectiveness analysis of seven pneumococcal vaccine in children. China Health Econ. 2013;32(4):71-5.

12. Zheng J, Cao L, Guo S, et al. Survey on the immunization status of category $B$ vaccine among children aged 1 to 2 years in China. Chinese $J$ Vaccin Immunization. 2012;18:233-7.

13. Gustafson R, Skowronski DM. Disparities in varicella vaccine coverage in the absence of public funding. Vaccine. 2005;23(27):3519-25.

14. Liao S, Huang T, Huang Y, et al. Survey of the status of self-paid varicella vaccination among children one to six years of age in Taiwan. J Microbiol Immunol Infect. 2007:40(2):112-5.

15. Manthiram K, Blood EA, Kuppuswamy $V$, et al. Predictors of optional immunization uptake in an urban south Indian population. Vaccine. 2014; 32(27):3417-23.

16. Wang Z, Wang W, Guo W, et al. Survey on vaccination rate of second class vaccine and influencial factors in children in rural areas of nine counties in Henan province. J Microbiol Immunol. 2013:3:53-61.

17. Chang J, Hou Z, Yue D, et al. Factors related to self-paid vaccination and its related factors among children aged $0-3$ years in China. Chin J Public Health. 2014;30(5):579-82.

18. Jiang $Y$, Yin $H$, Shi $Y H$, et al. Immunization status of extra EPI vaccines and its influencing factors among children aged 1-6 years in Chongqing. Chinese J Health Educ. 2013;29(7):605-7.

19. Tang $L$. The analysis of class II vaccination inoculation rates for $0-2$ year olds. Chinese J Clin. 2013;(5):43-5.

20. Liu XH. Shandong statistical yearbook. Beijing: China Statistics Press; 2014. from: http://www.stats-sd.gov.cn/tjnj/nj2014/indexch.htm

21. Zhang W, Zhang Y, Liu S, et al. Analysis of the current status of self-paid vaccination among children aged 1-5 in Shandong Province. J Mod Prev Med. 2016:10:048.

22. Wang C, Zhu L, Li JJ, et al. Vaccination status and influencing factors of children under five years old in Shandong province. Chinese J Child Health Care. 2015;23(11):1178-80.

23. Fang J, Xu L, Liu J. Analysis on vaccination status of 5 kinds of extra EPI vaccines in Jiangdong District. Mod Prev Med. 2012;39(17):4414-5.
24. Chen Q, Gao Z, Chen W, et al. Analysis on the immunization status of category $B$ vaccine among children aged 2 to 6 years in Tianjin. Mod Prev Med. 2014;41(014):2638-40

25. Wang Z. Study on inoculation state about the second type vaccines in Fengtai district of Beijing. Mod Prev Med. 2010;37(9):1662-3.

26. Cui FQ, Gofin R. Immunization coverage and its determinants in children aged 12-23 months in Gansu, China. Vaccine. 2007;25(4):664-71.

27. Chang J. Parents' willingness to pay and immunization service providers' willingness to provide of self-paid vaccines in three provinces of China. Shandong University Doctoral Thesis, Jinan, Shandong University; 2014.

28. Owais A, Hanif B, Siddiqui AR, et al. Does improving maternal knowledge of vaccines impact infant immunization rates? A community-based randomized-controlled trial in Karachi, Pakistan. BMC Public Health. 2011 ; 11(1):239

29. Wang LDL, Lam WWT, Wu JT, et al. Chinese immigrant parents' vaccination decision making for children: a qualitative analysis. BMC Public Health. 2014 14(1):133.

30. Allen JD, Coronado GD, Williams RS, et al. A systematic review of measures used in studies of human papillama virus (HPV) vaccine acceptability. Vaccine. 2010:28(24):4027-37.

31. Marlow LAV, Waller J, Wardle J. Parental attitudes to pre-pubertal HPV vaccination. Vaccine. 2007:25(11):1945-52

32. Ogilvie GS, Remple VP, Marra F, et al. Parental intention to have daughters receive the human papilloma virus vaccine. Can Med Assoc J. 2007:177(12):1506-12

33. Samad L, Tate AR, Dezateux C, et al. Differences in risk factors for partial and no immunization in the first year of life: prospective cohort study. BMJ. 2006;332(7553):1312-3

34. Itimi K, Dienye PO, Ordinioha B. Community participation and childhood immunization coverage: a comparative study of rural and urban communities of Bayelsa state, south-South Nigeria. Niger Med J. 2012;53(1):21.

35. Hou Z, Chang J, Yue D, et al. Determinants of willingness to pay for selfpaid vaccines in China. Vaccine. 2014;32(35):4471-7.

36. Marshall $H$, Ryan $P$, Roberton D. Uptake of varicella vaccine-a cross sectional survey of parental attitudes to nationally recommended but unfunded varicella immunization. Vaccine. 2005:23(46):5389-97.

37. Streng A, Seeger $K$, Grote V, et al. Varicella vaccination coverage in Bavaria (Germany) after general vaccine recommendation in 2004. Vaccine. 2010; 28(35):5738-45.

38. Santibanez TA, Santoli JM, Bridges CB, et al. Influenza vaccination coverage of children aged 6 to 23 months: the 2002-2003 and 2003-2004 influenza seasons. Pediatrics. 2006;118(3):1167-75.

39. Schuller KA, Probst JC. Factors associated with influenza vaccination among US children in 2008. J Infect Public Health. 2013;6(2):80-8.

40. Gauri V, Khaleghian P. Immunization in developing countries: its political and organizational determinants. World Dev. 2002;30(12):2109-32.

41. Li J. Gender inequality, family planning, and maternal and child care in a rural Chinese county. Soc Sci Med. 2004;59(4):695-708.

42. Dombkowski KJ, Lantz PM, Freed GL. The need for surveillance of delay in age-appropriate immunization. Am J Prev Med. 2002;23(1):36-42.
- fast, convenient online submission

- thorough peer review by experienced researchers in your field

- rapid publication on acceptance

- support for research data, including large and complex data types

- gold Open Access which fosters wider collaboration and increased citations

- maximum visibility for your research: over $100 \mathrm{M}$ website views per year

At BMC, research is always in progress.

Learn more biomedcentral.com/submissions 